\title{
Normative values for sleep parameters in pre-schoolers using actigraphy
}

\section{Sahlberg, Lassi}

2018-09

Sahlberg , L , Lapinleimu , H , Elovainio , M , Rönnlund , H \& Virtanen , I 2018 , ' Normative values for sleep parameters in pre-schoolers using actigraphy ', Clinical Neurophysiology , vol. 129 , no. 9 , pp. 1964-1970 . https://doi.org/10.1016/j.clinph.2018.06.027

http://hdl.handle.net/10138/311503

https://doi.org/10.1016/j.clinph.2018.06.027

cc_by_nc_nd

acceptedVersion

Downloaded from Helda, University of Helsinki institutional repository.

This is an electronic reprint of the original article.

This reprint may differ from the original in pagination and typographic detail.

Please cite the original version. 


\section{Normative values for sleep parameters in pre-schoolers using actigraphy}

Lassi Sahlberg, $\mathrm{MD}^{1,2,3}$, Helena Lapinleimu, $\mathrm{MD}, \mathrm{PhD}^{1,2}$, Marko Elovainio, prof ${ }^{4,5}$, Hanni Rönnlund, $\mathrm{MD}^{2,6}$, Irina Virtanen, $\mathrm{MD}, \mathrm{PhD}^{3}$

1 Department of Pediatrics and Adolescent Medicine, Turku University Hospital, Turku, Finland 2 Department of Pediatrics, University of Turku, Turku, Finland

3 Department of Clinical Neurophysiology, Turku University Hospital and University of Turku, Turku, Finland

4 Department of Psychology and Logopedics, University of Helsinki, Helsinki, Finland

5 National Institute for Health and Welfare, Helsinki, Finland

6 Health Care Center of Kaarina, Kaarina, Finland

Running head Actigraphy values of pre-schoolers' sleep

Word count 3326

Corresponding author and contact details

Irina Virtanen, $\mathrm{MD}, \mathrm{PhD}$

Department of Clinical Neurophysiology

Turku University Hospital

Kiinamyllynkatu 4-8

PL 52

20521 Turku

irina.virtanen@tyks.fi

Fax +35823133922

Tel +358405353071

Conflict of interest: None of the authors have potential conflicts of interest to be disclosed.

\section{Abstract}

\section{Objective}

There are currently no reference values for actigraphy-measured sleep length and fragmentation in preschool children. We created standardized parameters using a community sample. 


\section{Methods}

Ninety-seven 2-to-6-year-old children (56 boys) wore an actigraph on their non-dominant wrist for seven days. The data was extracted and scored, calculating total sleep time, sleep latency, sleep efficiency, fragmentation index, circadian rhythm length, cosine peak and light/dark ratio. Subjects were divided into groups of 2-3-year-olds, 4-5-year-olds and 6-year-olds. Means and standard deviations were calculated, and reference values were created using the $2.5^{\text {th }}$ and the $97.5^{\text {th }}$ percentiles.

\section{Results}

Reference intervals were 7 h 23 min - 9 h 47 min for 24-hour total sleep time, 0.2-48.4 minutes for sleep latency, 69-87 \% for sleep efficiency, 23-53\% for fragmentation index, 23 h 39 min - 24 h 24 min for circadian rhythm length, 12:37-15:53 for the timing of the cosine peak, and 1.14-5.63 for the light-dark ratio. With increasing age, daily sleep time, sleep latency, sleep fragmentation, and napping decreased.

\section{Conclusions}

We were able to create previously non-established reference values, including trends with increasing age, on actigraphy-assessed sleep in preschool children.

\section{Significance}

Sleep disorders in young children are easier to evaluate against normative data.

Keywords children, sleep, reference values, actigraphy, age

\section{Highlights}

We used actigraphy to create reference ranges for sleep parameters in 2-6-year-olds

Sleep time, latency, fragmentation and napping tend to decrease from 2 to 6 years

Individual circadian rhythm length is established in preschool age 


\section{Introduction}

Sleep disturbances are relatively common in children. Primary care providers have reported that approximately $20 \%$ of their 0-to-4-year-old patients had sleeping difficulties (Honaker et al. , 2016). The prevalence of manifest sleep problems reported by parents range between 11 and $12 \%$ (Honaker et al. , 2016), and at least one symptom of insomnia is reported in approximately $40 \%$ of children (Archbold et al. , 2002). The high prevalence of sleeping problems in children calls for reliable diagnostic tools for the clinicians. The current golden standard, the polysomnography (PSG) is an objective but a very demanding method of measuring sleep, and thus not always feasible.

Other approaches, such as parent-filled sleep diaries or questionnaires, create poor estimates of nocturnal sleep because they do not delineate sleep architecture (Werner et al. , 2008, Lam et al. , 2011). Sleep may be fragmented, superficial, or inefficient, and questionnaires are usually not sensitive enough to spot nocturnal awakenings (Iwasaki et al. , 2010, Simard et al. , 2013).

Furthermore, parents tend to overestimate sleep disturbances in their children when their own sleep is impaired (Rönnlund et al. , 2016).

Actigraphy has been utilized in sleep research for over forty years (Reite et al. , 1995), and it has become a more common method of studying sleep than PSG (Sadeh, 2011). A small wrist-worn actigraph is cost-effective, unobtrusive, and accessible as compared to PSG. However, an actigraph only measures movement and therefore it only indicates quiet, non-moving phases that can be interpreted as sleep, being unable to distinguish between different stages of sleep. When compared to adults, children tend to move more in their sleep (De Koninck et al. , 1992) which may explain why actigraphy systematically underestimates their total sleep time and sleep efficiency in contrast to PSG regardless of the used device (Spruyt et al. , 2011, Meltzer et al. , 2012b, Belanger et al. , 2013, Waldon et al. , 2016). Despite the large amount of devices and software, standardized sleep parameters for actigraphy are missing due to different mathematical backgrounds and validity of the algorithms (te Lindert and Van Someren, 2013, Ancoli-Israel et al. , 2015). Several attempts to generate better-fitting algorithms for children have been reported with variable success (Meltzer et al. , 2011, Meltzer et al. , 2015b).

The goal of this study was to calculate sleep parameters in younger children using actigraphy and to create age-specific reference values for these parameters. We used a micro-electronic-mechanical- 
system (MEMS) actigraph to assess sleep parameters in 2-to-6-year-old children, and to establish the age-specific 95 per cent reference ranges for each parameter.

\section{Methods}

\subsection{Participants}

The participants of this study were 2-6-year-old Finnish-speaking Caucasian children living with their biological parents and attending regular daycare. They acted as the non-adopted controls in the FinAdo 2 study, which is a follow-up study on the health and wellbeing of international adoptees in Finland as compared to the original population. Through giving a total of 1560 invitational letters to be handed out in 16 daycare centres in the cities of Turku and Kaarina, Southwest Finland, we recruited 117 subjects. After initial consent, seven subjects were excluded because they moved to another city or could not be contacted. Two subjects were internationally adopted and therefore were not included in the control group. After extraction of recorded actigraphy data, eleven registrations failed to meet the inclusion criteria of at least four nights of data due to missing or detached bracelet or mechanical malfunction, and were excluded. Data from 97 registrations was finally included in the analysis. The registrations were carried out between January 2014 and February 2015.

\subsection{Procedures}

After accepting the invitation all participating families were contacted via telephone and two meetings were settled: one for attaching the actigraphy bracelet and giving instructions and questionnaires on the medical history, sleep disturbances, and socioeconomic status of the family, and another for extracting the bracelet and debriefing. The meetings were arranged at the Department of Clinical Neurophysiology, Turku University Hospital, Finland. The actigraphy bracelet was attached to the non-dominant wrist of the child and a sleep diary and a set of questionnaires were given to the family (Rönnlund et al. , 2016). Parents and day care centre staff were advised to press "bed time" on a button on the bracelet when the child went to bed with the intention of sleeping and to press "get up time" when the child got out of bed, including nap times. They were also prompted to write down every bed time, get up time, time of removal, and time of reattachment of the bracelet in a sleep diary. The day care centre staff were further advised to mark routine start and end times for daytime napping in the sleep diaries. After seven days, the family returned to the study centre where a study assistant removed the bracelet, collected the 
questionnaires, extracted the actigraphy data and confirmed that at least four nights of data had been recorded.

\subsection{Actigraphy}

The actigraph used in this study was a MEMS based accelerometer (GeneActiv Original, Activinsights Ltd, Kimbolton, UK) originally chosen for the adoptee leg of the FinAdo study. It has a range of $8 \mathrm{G}$ with a resolution of $3.8 \mathrm{mG}$, with a triaxial sensor recording both motional and gravitational acceleration and it has been validated for the assessment of physical activity in children (Phillips et al. , 2013). The data sampling rate was $50 \mathrm{~Hz}$. Due to the lack of GeneActiv software-calculated parameters, the Actiwatch Activity \& Sleep analysis 7 version 7.31 (AAS7) software with medium sensitivity and a 1-minute epoch length was used to interpret the data. The $\mathrm{X}$-axis movement data was converted to activity counts. A 1st order butterworth IIR filter with a 3$11 \mathrm{~Hz}$ bandwidth was applied to the data, and the highest acceleration value of each 1 second epoch was calculated and summed over $30 \mathrm{~s}$ epochs. The sum was then defined as G. To obtain Actiwatch $30 \mathrm{~s}$ epoch data (A), the equation $\mathrm{A}=49 \mathrm{G}-23$ was used (Virkkala, 2012). The epochs were scored as sleep or wake by the AAS7 by using an activity count threshold of 40 per minute, i.e. an activity count of less than 40 during one minute of registration was designated as immobility suggestive of sleep. Sleep start was defined by at least ten minutes of consecutively recorded immobile data, with no more than 1 epoch of movement within that time, following bedtime. The sleep end algorithm calculated backwards from the get up time for a similar consecutive period of immobility, allowing for up to two epochs of movement within the period.

A successful conversion and validation of GeneActiv actigraphy raw data to Actiwatch activity counts has also previously been reported; the GeneActiv data was scored almost identically as sleep/wake epochs using Actiwatch software compared with Actiwatch-L obtained activity counts (te Lindert and Van Someren, 2013). By applying this method, the GeneActiv sleep/wake detection accuracy can be approximated from previous Actiwatch-across-PSG validation studies in similar age groups. A validation study of the epoch-by-epoch ability of AAS7 with medium sensitivity to detect sleep as compared to PSG defined a moderate specificity, 69.9\% (SD 16.4\%), but a high sensitivity, 92.7\% (SD 2.7\%), in the 2-5-year-old population (Belanger et al. , 2013). High intra class correlation (ICC) was found between a wrist-attached AAS7 with medium sensitivity and PSG in sleep time (0.94), sleep latency (0.92) and sleep efficiency (0.73). However, a low ICC in number of awakenings (0.42) was reported (Meltzer et al. , 2015a) 


\subsection{Parameters}

Bed time and getting up time were defined by the actigraph button-press time and / or the sleep diaries. If there was no registered button-press, the sleep diaries were used. All data were double checked for consistency. Seven parameters of sleep were included in the reference values: total sleep time (total time of epochs defined as sleep) in hours and minutes, sleep latency from bed time to sleep start, fragmentation index (the sum of the percentage of time in bed spent moving and the percentage of immobility phases shorter than one minute out of all immobility phases; a measure of nocturnal restlessness), sleep efficiency (the percentage of time spent asleep whilst in bed), circadian rhythm (intrinsic circadian period length from a periodogram using 1-minute resolution; usually slightly over 24 hours in all humans), cosine peak (timing of peak daytime activity, a measure of the timing of a person's most active period during 24 hours), and light to dark ratio (total activity count between 6 am and $6 \mathrm{pm}$ divided by total activity count between $6 \mathrm{pm}$ and 6 am; low values indicate more wake activity during the night-time hours). Values for total sleep time, sleep latency, fragmentation index, and sleep efficiency were calculated for both nocturnal and 24hour sleep and averaged across seven days.

\subsection{Statistical analysis}

Subjects were divided into three groups based on developmental similarity of sleep structure (Sheldon, 2014): 2-3-year-olds, 4-5-year-olds and 6-year-olds. Mean and standard deviation (SD) were calculated for each parameter. Reference values were created using the $2.5^{\text {th }}$ percentile parameter (mean $-1.96 * \mathrm{SD})$ and the $97.5^{\text {th }}$ percentile (mean $\left.+1.96 * \mathrm{SD}\right)$. Group differences were evaluated using the nonparametric Kruskall-Wallis test and post-hoc comparisons made using the Wilcoxon signed rank test with Bonferroni correction. Calculations were carried out using SAS version 9.4., and SPSS ver. 24. All data is presented as mean (SD) or N (\%).

\subsection{Ethics}

The study was approved by the Ethics review committee of the Hospital District of Southwest Finland and all the parents gave a written informed consent.

\section{Results}

Table 1 shows the participant characteristics. Thirteen children had at least one chronic disease: asthma $(\mathrm{N}=9)$, atopy $(\mathrm{N}=3)$, allergies $(\mathrm{N}=1)$, or hypothyreosis treated with thyroxin and euthyreotic at the time of the study $(\mathrm{N}=1)$. During the registration, fourteen children were receiving at least one 
medication: thyroxin $(\mathrm{N}=1)$, an inhaled corticosteroid $(\mathrm{N}=3)$, a systemic antibiotic $(\mathrm{N}=3)$, a local antibiotic $(\mathrm{N}=1)$, an anti-inflammatory drug $(\mathrm{N}=3)$, a nasal decongestant $(\mathrm{N}=2)$, a first generation antihistamine $(\mathrm{N}=1)$, or a second-generation antihistamine $(\mathrm{N}=4)$. None of the children had a diagnosed sleep disorder, nor did they receive melatonin or other sleep-enhancing medication during the study. The unemployment rate of the parents as well as the age distribution of the families in this study matched the 2014 national average (Official Statistics of Finland, 2015). No data of the percentage with which the bracelet button was used to indicate bed time or get up time was calculated, but as the parents and the day care centre staff were prompted to write down these times, they could be easily tracked down in almost every case, and trends in movements could be used as cues for bed times and get up times in the rare cases with no objective indicators.

The 24-hour time in bed varied between 8 h 23 min and 12 h 32 min (mean 11 h 21 min (48 min)) in 2-3-year-olds, between 9 h 51 min and 12 h 31 min (mean 11 h 0 min (36 min)) in 4-5-year-olds, and between $9 \mathrm{~h} 18 \mathrm{~min}$ and $11 \mathrm{~h} 11 \mathrm{~min}$ (mean $10 \mathrm{~h} 27 \mathrm{~min}(30 \mathrm{~min})$ ) in 6-year-olds.

In between-groups comparisons, overnight sleep efficiency was higher $(\mathrm{p}=0.009)$ while sleep latency was shorter and fragmentation index lower ( $\mathrm{p}=0.036$ and 0.018 , respectively) in older age groups. When averaged over 24 hours, total sleep time was shorter and fragmentation index was lower ( $\mathrm{p}=0.029$ and $\mathrm{p}=0.039$, respectively) while sleep efficiency was higher $(\mathrm{p}=0.003)$ and sleep latency tended to shorten in older age groups $(\mathrm{p}=0.052)$. Furthermore, light to dark ratio was higher in older age groups $(\mathrm{p}=0.006)$. In post-hoc group-wise comparisons, however, these differences disappeared. (Figures 1 and 2)

For the whole group, the $95 \%$ reference range was 7 h 23 min - 9 h 47 min for 24-hour total sleep time, 0.2-48.4 min for sleep latency, 69-87\% for sleep efficiency, 23-53\% for fragmentation index, 23 h 39 min - 24 h 24 min for circadian rhythm length, 12:37-15:53 for the timing of the cosine peak, and 1.14-5.63 for the light-dark ratio.

The created normative sleep parameters are presented in Table 2. The length of the 24 -hour sleep time and the differences between means of 24 -hour sleep and nocturnal sleep (41 min, 16 min, 6 min) decreased with age. Nocturnal sleep time was shortest in the youngest age group. 6-year-olds had the smallest coefficient of variation $(\mathrm{CV})$ in nocturnal sleep $(6.58 \%, 6.85 \%, 5.05 \%)$. The CV 
in 24 -hour sleep decreased with age (8.35\%, 6.25\%, $4.79 \%)$. In 2-3-year-olds, 35 out of 36 children napped on a mean of 4.1 (1.8) days, in 4-5-year-olds, 34 out of 42 children napped on a mean of 2.9 (1.6) days, and in 6-year-olds, 10 out of 20 children napped on a mean of 2.6 (1.8) days.

The mean daily sleep latency in 2-3-year-olds was 3.8 min longer than in 4-5-year-olds and 9.6 minutes longer than in 6-year-olds. Fragmentation index was smallest in 6-year-olds (34.2 (8.8) \%) while the two remaining groups had almost identical means: 39.0 (7.2) \% in 2-3-years-olds and 38.1 (7.1) $\%$ in 4-5-year-olds. The 24-hour sleep efficiency increased by $1.4 \%$ between 2 -3-years-olds and 4-5-year-olds and a further $1.9 \%$ as compared to 6-year-olds. The circadian rhythm was similar between groups. Cosine peak values followed no trends when compared between the age groups. Light to dark ratio increased by $6.9 \%$ between 2-3-year-olds and 4-5-year-olds and $21.1 \%$ between 4-5-year-olds and 6-year-olds. CV was the smallest in 6-year-olds (32.7 \%, 37.1 \%, $24.9 \%)$. No gender differences were found in any index between any age groups.

\section{Discussion}

The purpose of this study was to create reference values for sleep parameters for 2-6-year-old children using actigraphy. We estimated a reference range of 7 h 23 min - 9 h 47 min for 24-hour total sleep time in the group with varying sleep latencies of up to 48 minutes and a fairly low sleep efficiency of 69-87\%. Circadian rhythm fell close to previously reported normal values for adults (Czeisler et al. , 1999), supporting the idea of an early development of a natural sleep/wake cycle, and the timing of the circadian rhythm was consistent between two and six years of age. Nocturnal sleep time, naps, and sleep latency shortened between the ages of two and six, and a decrease in napping in the older age groups was verified. The smallest variance of sleep time in the six-year-old group suggests that the overall variance of sleep time diminishes between the years of two to six. The decrease in fragmentation index points to lower levels of nocturnal motor restlessness in older age groups in preschool children.

In a previous PSG study among 153 3-5-year-old healthy children the average sleep time was 7.9 (0.7) hours and the range of sleep time was 5.4-9.3 hours. Sleep efficiency was 90.0 (7.0) \% and sleep latency was 24.1 (25.6) minutes (Montgomery-Downs et al. , 2006). Compared to our data, their reported sleep times were shorter but sleep latencies similar, furthermore, the sleep efficiencies were significantly higher than in our study. A systematic review of diary and questionnaire obtained sleep parameters estimated the mean of 24 hours sleep time to be 12.0 hours in the 2-3-year-old 
group and 11.5 hours in the 4-5-year-old group (Galland et al. , 2012), which is clearly much longer than what we observed. PSG electrodes and the foreign environment alter the quantitative sleep parameters (Agnew et al. , 1966), which may be reflected in the fairly short mean and a large range of sleep time in the PSG studies (Montgomery-Downs et al. , 2006). Questionnaires and diaries, on the other hand, seem to overestimate sleep time, (Iwasaki et al. ,2010, Lam et al. , 2011, Galland et al. , 2012, Simard et al. , 2013), and parental sleep problems, on the other hand, cause the parent to report more sleep related problems in their offspring (Rönnlund et al. , 2016). Previous actigraphy data on children is scarce and mainly focuses on total sleep time and sleep latency, giving mean nocturnal sleep time estimates between 7.5 and 8.5 hours and mean sleep latencies between 25 and 45 minutes in two-to-six-year-old children (Lam et al. , 2011, Spruyt et al. , 2011, Simard et al. , 2013), which is fairly consistent with our results.

Fragmentation index was also calculated in this study. It has been shown to increase significantly in children with obstructive sleep apnoea as compared to primary snorers (O'Driscoll et al. , 2010). Furthermore, it has been shown to be an excellent tool in estimating the effectiveness of therapeutic interventions in adult patients with obstructive sleep apnoea syndrome (Aubert-Tulkens et al. , 1987). Studies evaluating the significance of the fragmentation index in children are scarce, although its ability to depict the restlessness of sleep could be utilized in sleep medicine in the future.

As parent-reported sleep related problems are common, it is important to first establish normal actigraphy measured sleep parameters and this was achieved through analysing the data of 97 subjects. While there are earlier studies in which actigraphy-assessed nocturnal sleep has been recorded, there are no reported standard values on actigraphy measured sleep with confidence intervals derived from a general population of two-to-six-year-old children. These parameters provide novel tools for the clinician assessing the severity of sleep problems in a child.

The strengths of this study include the low number of excluded registrations and the sparseness of dropouts. The actigraph recorded at least the recommended five days (Acebo et al. , 1999) in all but two included subjects, and even in these two, the four days of recorded data was very consistent. The number of participants was satisfactory despite the low recruitment rate. The participating families represented the national average by their age and unemployment rate (Official Statistics of Finland, 2015, Rönnlund et al. , 2016). The sleep scoring logarithm used in this study is validated and has a high sensitivity of $92.7 \%$ (SD 2.7\%) (Belanger et al. , 2013). We also included children 
with common chronic childhood illnesses such as asthma and atopy, which increases the generalizability of our results.

\subsection{Study limitations}

There are some limitations in this study. First, the specificity of the Actiwatch 40 logarithm is only moderate $(69.9 \%)$. The moderate specificity means that the scoring of wakefulness is only moderately correct (Meltzer et al. , 2012a, Belanger et al. , 2013), because the actigraph cannot determine between lying still awake in bed and sleeping. However, these logarithms have been calculated in adult populations. Pre-schoolers rarely stay still in bed when awake, which reduces the risk of overestimating sleep time in this population. On the other hand it has been shown that young children tend to move more than adults in their sleep (De Koninck et al. , 1992) and therefore actigraphy possibly overestimates wake after sleep onset, underestimates total sleep time and underestimates sleep efficiency in children. This probably explains the disparity of our sleep efficiencies as compared to the earlier PSG studies (Montgomery-Downs et al. , 2006). In fact, in a validation study the Actiwatch 40 logarithm, against which our actigraphs have been validated, underestimated mean total sleep time by 25 minutes and sleep efficiency by $3.9 \%$ (Belanger et al. , 2013). This might partly explain the disparity between our results and the recent sleep recommendations by the National Sleep Foundation recommending 11-14 hours of sleep to 1-2 year olds and 10-13 hours to 3-5 year olds. Also the article in question acknowledges the bias toward longer sleep durations as most of the literature on sleep duration is gathered using subjective methods. These methods do not allow differentiating between actual sleep time and time in bed as the latter is always longer in duration (Hirshkowitz et al. , 2015). Further, the approach of converting MEMS-gathered raw data into activity counts is relatively new and has only fairly recently started to gain ground as a method of sampling data (te Lindert and Van Someren, 2013). What comes to our study population, it was an ethnically and linguistically homogeneous group of children attending regular day care, and therefore, the generalizability of the results to all populations cannot be guaranteed. Especially day napping may be an issue when defining sleep trends in preschool children. Our cohort was invited by sending information leaflets to municipal day care centres, which have a very homogeneous way of working in Finland. Practically all children, with the possible exception of 6-year-olds, attend a 30-minute to 2-hour day nap or quiet moment after lunch, between 12 noon and 2 p.m. Younger children can sleep for a longer period of time, but sleeping is not required and older children are generally allowed to rise after 30 minutes, if they do not fall asleep, therefore allowing for the natural tendency of each child to stop napping at his or her preferred age. 


\section{Conclusions}

Our study created quantitative activity-based parameters of sleep and we were able to describe their development with age in two-to-six-year-old children. Even though these reference values cannot depict the structural sleep parameters, they enable the possibility of objectively estimating the amount and efficiency of a child's sleep. Advances in technology have made gathering data to a compact form easier that is resulting in new MEMS actigraphs getting common in actigraphy studies. It is important to continue the validation of the systems that convert the high amount of collected raw data to actual sleep parameters.

\section{Acknowledgements}

We thank research assistants Anniina Mäkinen, Miia Salokannel and Sergei Ionin for their work in executing the actigraph registrations; and the Department of Clinical Neurophysiology at Turku University Hospital for supplying the facilities for the actigraph registrations. We thank Jaakko Matomäki for designing the analysis methods and carrying out the statistical analyses. The study was financially supported by the Foundation for Pediatric Research, Signe and Ane Gyllenberg Foundation and the research funding of the Hospital District of Southwest Finland. None of the funding sources have attributed any coworkmanship in any part of the study. 


\section{References}

Acebo C, Sadeh A, Seifer R, Tzischinsky O, Wolfson A, Hafer A, et al. Estimating sleep patterns with activity monitoring in children and adolescents: how many nights are necessary for reliable measures? Sleep. 1999;22:95-103.

Agnew H, Webb W, Williams R. The first night effect: an EEG study of sleep. Psychophysiology. 1966;2:263-6.

Ancoli-Israel S, Martin J, Blackwell T, Buenaver L, Liu L, Meltzer L, et al. The SBSM Guide to Actigraphy Monitoring: Clinical and Research Applications. Behav Sleep Med. 2015;13 Suppl 1:S4-S38.

Archbold K, Pituch K, Panahi P, Chervin R. Symptoms of sleep disturbances among children at two general pediatric clinics. J Pediatrics. 2002;140:97-102.

Aubert-Tulkens G, Culee C, Harmant-Van Rijckevorsel K, Rodenstein D. Ambulatory evaluation of sleep disturbance and therapeutic effects in sleep apnea syndrome by wrist activity monitoring. Am Rev Resp Dis. 1987;136:851-6.

Belanger M, Bernier A, Paquet J, Simard V, Carrier J. Validating actigraphy as a measure of sleep for preschool children. J Clin Sleep Med. 2013;9:701-6.

Czeisler C, Duffy J, Shanahan T, Brown E, Mitchell J, Rimmer D, et al. Stability, precision, and near-24-hour period of the human circadian pacemaker. Science. 1999;284:2177-81.

De Koninck J, Lorrain D, Gagnon P. Sleep positions and position shifts in five age groups: an ontogenetic picture. Sleep. 1992;15:143-9.

Galland B, Taylor B, Elder D, Herbison P. Normal sleep patterns in infants and children: a systematic review of observational studies. Sleep Med Rev. 2012;16:213-22.

Hirshkowitz M, Whiton K, Albert S, Alessi C, Bruni O, DonCarlos L, et al. National Sleep Foundation's sleep time duration recommendations: methodology and results summary. Sleep health. 2015;1:40-3.

Honaker S, Meltzer LJ. Sleep in pediatric primary care: A review of the literature. Sleep Med Rev. 2016;25:31-9.

Iwasaki M, Iwata S, Iemura A, Yamashita N, Tomino Y, Anme T, et al. Utility of subjective sleep assessment tools for healthy preschool children: a comparative study between sleep logs, questionnaires, and actigraphy. J Epidemiol. 2010;20:143-9.

Lam J, Mahone E, Mason T, Scharf S. Defining the roles of actigraphy and parent logs for assessing sleep variables in preschool children. Behav Sleep Med. 2011;9:184-93.

Meltzer L, Hiruma L, Avis K, Montgomery-Downs H, Valentin J. Comparison of a Commercial Accelerometer with Polysomnography and Actigraphy in Children and Adolescents. Sleep. 
2015a;38:1323-30.

Meltzer L, Montgomery-Downs H, Insana S, Walsh C. Use of actigraphy for assessment in pediatric sleep research. Sleep Med Rev. 2012a;16:463-75.

Meltzer L, Walsh C, Peightal A. Comparison of actigraphy immobility rules with polysomnographic sleep onset latency in children and adolescents. Sleep Breath. 2015b;19:1415-23.

Meltzer L, Walsh C, Traylor J, Westin A. Direct comparison of two new actigraphs and polysomnography in children and adolescents. Sleep. 2012b;35:159-66.

Meltzer L, Westin A. A comparison of actigraphy scoring rules used in pediatric research. Sleep Med. 2011;12:793-6.

Montgomery-Downs H, O'Brien L, Gulliver T, Gozal D. Polysomnographic characteristics in normal preschool and early school-aged children. Pediatrics. 2006;117:741-53.

O'Driscoll D, Foster A, Davey M, Nixon G, Horne R. Can actigraphy measure sleep fragmentation in children? Arch Dis Child. 2010;95:1031-3.

Official Statistics of Finland. Employment and unemployment 2014: Labour force survey [epublication]. 2015.

Phillips L, Parfitt G, Rowlands A. Calibration of the GENEA accelerometer for assessment of physical activity intensity in children. J Sci Med Sport. 2013;16:124-8.

Reite M, Buysse D, Reynolds C, Mendelson W. The use of polysomnography in the evaluation of insomnia. Sleep. 1995;18:58-70.

Rönnlund H, Elovainio M, Virtanen I, Matomäki J, Lapinleimu H. Poor Parental Sleep and the Reported Sleep Quality of Their Children. Pediatrics. 2016;137.

Sadeh A. The role and validity of actigraphy in sleep medicine: an update. Sleep Med Rev. 2011;15:259-67.

Sheldon S. Development of sleep in infants and children. In: Sheldon S, Ferber R, Kryger M, Gozal D, editors. Principles and practice of pediatric sleep medicine. Elsevier; 2014.

Simard V, Bernier A, Belanger M, Carrier J. Infant attachment and toddlers' sleep assessed by maternal reports and actigraphy: different measurement methods yield different relations. J Ped Psychol. 2013;38:473-83.

Spruyt K, Gozal D, Dayyat E, Roman A, Molfese D. Sleep assessments in healthy school-aged children using actigraphy: concordance with polysomnography. J Sleep Res. 2011;20:223-32. te Lindert B, Van Someren E. Sleep estimates using microelectromechanical systems (MEMS). Sleep. 2013;36:781-9.

Waldon J, Begum E, Gendron M, Rusak B, Andreou P, Rajda M, et al. Concordance of actigraphy with polysomnography in children with and without attention-deficit/hyperactivity disorder. J Sleep 
Res. 2016;25:524-33.

Werner H, Molinari L, Guyer C, Jenni O. Agreement rates between actigraphy, diary, and questionnaire for children's sleep patterns. Arch Ped \& Adol Med. 2008;162:350-8.

Virkkala J. Using Accelerometers as actigraphs. J Sleep Res. 2012;21:198-9. 


\section{Legends}

Figure 1. Box plots of total sleep time and sleep latency distributions across age groups, over 24 hours and overnight. Median / quartile / range.

Figure 2. Box plots of sleep efficiency and fragmentation index distributions across age groups, over 24 hours and overnight. Median / quartile / range. 
Table 1. Characteristics of the study participants

\begin{tabular}{|c|c|}
\hline \multicolumn{2}{|l|}{ Demographic characteristics } \\
\hline Sample (n/\%) & $97(100)$ \\
\hline Male $(n / \%)$ & $52(53.6)$ \\
\hline Age (mean/SD) & $4.7(1.3)$ \\
\hline $2 \mathrm{yrs}(\mathrm{n} / \%)$ & $11(11.3)$ \\
\hline 3 yrs (n/\%) & $24(24.7)$ \\
\hline $4 \mathrm{yrs}(\mathrm{n} / \%)$ & $18(18.6)$ \\
\hline 5 yrs $(n / \%)$ & $24(24.7)$ \\
\hline $6 \mathrm{yrs}(\mathrm{n} / \%)$ & $20(20.6)$ \\
\hline \multicolumn{2}{|l|}{ Combined study age groups } \\
\hline $2-3 \mathrm{yrs}(\mathrm{n} / \%)$ & $35(36.1)$ \\
\hline $4-5$ yrs $(n / \%)$ & $42(43.3)$ \\
\hline $6 \mathrm{yrs}(\mathrm{n} / \%)$ & $20(20.6)$ \\
\hline Room sharing with parents ( $\mathrm{n} / \%)$ & $20(20.6)$ \\
\hline Number of siblings (mean/SD) & $0.9(1.1)$ \\
\hline Summer/Winter during registration (n/\%) & $52(53.6) / 45(46.4)$ \\
\hline \multicolumn{2}{|l|}{ Number of successfully recorded nights ( $\mathrm{n} / \%$ ) } \\
\hline 4 & $2(2.1)$ \\
\hline 5 & $1(1.0)$ \\
\hline 6 & $8(8.2)$ \\
\hline 7 & $87(89.7)$ \\
\hline Mothers $(n)$ & 97 \\
\hline Age (mean/SD) & $32.7(6.1)$ \\
\hline \multicolumn{2}{|l|}{ Education } \\
\hline Lower secondary school (n/\%) & $1(1.0)$ \\
\hline Upper secondary school (n/\%) & $3(3.1)$ \\
\hline Trade school $(\mathrm{n} / \%)$ & $13(13.4)$ \\
\hline Postsecondary vocational education ( $\mathrm{n} / \%$ ) & $25(25.8)$ \\
\hline University (n/\%) & $50(51.5)$ \\
\hline Fathers $(n)$ & 91 \\
\hline Age (mean/SD) & $39.0(10.1)$ \\
\hline \multicolumn{2}{|l|}{ Education } \\
\hline Lower secondary school (n/\%) & $3(3.3)$ \\
\hline Upper secondary school (n/\%) & $5(5.5)$ \\
\hline Trade school $(\mathrm{n} / \%)$ & $25(27.5)$ \\
\hline Postsecondary vocational education (n/\%) & $15(16.5)$ \\
\hline University $(\mathrm{n} / \%)$ & $40(44.0)$ \\
\hline
\end{tabular}


Table 2 Normative values for sleep parameters across groups

\begin{tabular}{|c|c|c|c|c|c|c|c|c|c|c|c|c|}
\hline & \multicolumn{4}{|c|}{2 to 3 years } & \multicolumn{4}{|c|}{4 to 5 years } & \multicolumn{4}{|c|}{ 6+years } \\
\hline & Mean & SD & $2.5 \%$ & $97.5 \%$ & Mean & SD & $2.5 \%$ & $97.5 \%$ & M ean & SD & $2.5 \%$ & $97.5 \%$ \\
\hline \multicolumn{13}{|l|}{ Total sleep time (hh:mm) } \\
\hline 24 hours & $8: 47$ & $0: 44$ & $7: 20$ & $10: 12$ & $8: 32$ & $0: 32$ & $7: 29$ & $9: 35$ & $8: 21$ & $0: 24$ & $7: 34$ & 9:08 \\
\hline Night & 8:06 & $0: 32$ & 7:03 & 9:08 & $8: 16$ & $0: 34$ & $7: 10$ & $9: 22$ & $8: 15$ & $0: 25$ & $7: 26$ & 9:05 \\
\hline \multicolumn{13}{|l|}{ Sleep latency (minutes) } \\
\hline 24 hours & 27.9 & 14.3 & 0.0 & 55.9 & 24.1 & 11.2 & 2.2 & 46.0 & 18.3 & 7.9 & 2.9 & 33.7 \\
\hline Night & 27.8 & 13.7 & 0.8 & 54.7 & 23.9 & 11.5 & 1.3 & 46.5 & 18.0 & 8.3 & 1.9 & 34.2 \\
\hline \multicolumn{13}{|l|}{ Fragmentation index (\%) } \\
\hline 24 hours & 39.0 & 7.2 & 24.9 & 53.1 & 38.1 & 7.1 & 24.2 & 51.9 & 34.2 & 8.8 & 17.0 & 51.4 \\
\hline Night & 39.9 & 7.4 & 25.4 & 54.5 & 38.5 & 7.4 & 23.9 & 53.1 & 34.1 & 8.8 & 16.9 & 51.4 \\
\hline \multicolumn{13}{|l|}{ Sleep efficiency (\%) } \\
\hline 24 hours & 76.9 & 3.5 & 70.0 & 83.8 & 78.3 & 3.6 & 71.2 & 85.5 & 80.2 & 4.3 & 71.7 & 88.7 \\
\hline Night & 77.8 & 3.7 & 70.6 & 85.1 & 79.0 & 3.8 & 71.5 & 86.5 & 80.7 & 4.5 & 71.9 & 89.5 \\
\hline Circadian rhythm (hh:mm) & $24: 01$ & $0: 12$ & $23: 37$ & $24: 24$ & $24: 03$ & $0: 09$ & $23: 46$ & $24: 20$ & $24: 00$ & $0: 15$ & $23: 31$ & $24: 30$ \\
\hline Cosine peak (hh:mm) & $14: 16$ & $0: 47$ & $12: 44$ & $15: 48$ & $14: 24$ & $0: 54$ & $12: 39$ & $16: 09$ & $13: 54$ & $0: 43$ & $12: 29$ & $15: 18$ \\
\hline Light/Dark ratio & 3.12 & 1.02 & 1.13 & 5.12 & 3.31 & 1.23 & 0.91 & 5.71 & 4.01 & 1.00 & 2.06 & 5.97 \\
\hline
\end{tabular}

